Artigo Original

\title{
Padrão de co-ativação dos músculos do tronco durante exercícios com haste oscilatória
}

\author{
Nise Ribeiro Marques ${ }^{1,2}$ \\ Camilla Zamfolini Hallal ${ }^{1,2}$ \\ Mauro Gonçalves ${ }^{1,2}$ \\ ${ }^{1}$ Faculdade de Ciências e Tecnologia, UNESP - Univ Estadual Paulista, \\ Campus de Presidente Prudente, SP, Brasil \\ ${ }^{2}$ Laboratório de Biomecânica, Departamento de Educação Física, Instituto de Biociências, \\ UNESP - Univ Estadual Paulista, Rio Claro, SP, Brasil
}

\begin{abstract}
Resumo: Este estudo objetivou comparar o padrão de co-ativação dos músculos do tronco durante exercícios com uma haste oscilatória. Participaram do estudo doze voluntárias, que realizaram três diferentes exercícios. A atividade eletromiográfica foi coletada dos músculos oblíquo interno (OI), oblíquo externo (OE), reto abdominal (RA), multifido (MU) e iliocostal lombar (IL). O sinal de eletromiografia (EMG) foi analisado no domínio do tempo (RMS) e foram calculadas as razões: ântero-posterior $(\mathrm{A} / \mathrm{P}=\mathrm{RA}+\mathrm{OE}+\mathrm{OI} / \mathrm{MU}+\mathrm{IL}), \mathrm{MU} / \mathrm{IL}$ e $\mathrm{OE} / \mathrm{OI}$. A oscilação bilateral da haste no plano frontal (exercício II) provocou maior valor da razão MU/IL que a oscilação unilateral da haste no plano sagital (exercício III). Já a oscilação bilateral da haste no plano frontal (exercício II) e a oscilação unilateral da haste no plano sagital (exercício III) geraram maior valor de OI/OE que a oscilação bilateral da haste no plano transverso (exercício I). Portanto, os exercícios II e III exigem maior ativação de músculos estabilizadores do tronco, sendo mais indicados para treinamentos que visem o maior recrutamento destes músculos nas atividades de vida diária.
\end{abstract}

Palavras-chave: biomecânica; eletromiografia; exercício.

\section{Trunk muscles co-activation patterns during exercises with oscillatory pole}

\begin{abstract}
This study aimed to compare trunk muscle co-activation pattern during exercises using an oscillatory pole. Twelve volunteers participated in this study, in which they performed three different exercises. EMG activity of internal oblique (IO), external oblique (EO), rectus abdominis (RA), multifidus (MU) and iliocostalis lumborum (IL) was collected. The EMG signals were analyzed in time domain (RMS) and muscles activation ratios were computed as follow: anterior-posterior (A/P=RA+EO+IO/MU+IL), MU/IL and $\mathrm{OE} / \mathrm{OI}$. The bilateral oscillation of the pole in frontal plane (exercise II) promoted a higher value of MU/IL ratio than unilateral oscillation of the pole in sagital plane (exercise III). Also, the bilateral oscillation of the pole in frontal plane (exercise II) and the unilateral oscillation of the pole in sagital plane (exercise III) caused higher values of the IO/EO than bilateral oscillation of the pole in transversal plane (exercise I). Thus, the exercises II and III required higher activation of trunk stabilizer muscles, being more indicate for training, which aims higher recruitment of these muscles in daily activities.
\end{abstract}

Keywords: biomechanics; electromyography; exercise.

\section{Introdução}

A estabilidade da coluna lombar é mantida por meio da ação integrada de três subsistemas: o ativo, o passivo e o neural (PANJABI, 1992; GRANATA \& MARRAS, 2000). O subsistema ativo é o primeiro mecanismo acionado para a estabilização dos segmentos da coluna frente às perturbações externas (NEWCOMER et al., 2002; MACDONALD, MOSELEY \& HODGES, 2009). Esse subsistema é composto pelos músculos do tronco, que podem ser classificados em locais e globais de acordo com suas características anatômicas e funcionais. Os músculos locais possuem inserção nas vértebras lombares e, assim, estabilizam os segmentos vertebrais. Já, os músculos globais possuem inserções em pontos anatômicos diferentes das vértebras e apresentam a função de gerar movimentos de grande amplitude e torque (O'SULLIVAN et al., 1997; O'SULLIVAN et al., 2002; OLIVEIRA \& GONÇALVES, 2009).

Contudo, estudos recentes têm mostrado que a direção das forças de perturbação externa é o principal fator que irá determinar quais músculos do tronco serão recrutados para a estabilização da coluna (VAN DIEEN, CHOLEWICKI \& RADEBOLD, 2003; REEVES, NARENDRA \& CHOLEWICKI, 2007). Assim, para que haja 
estabilidade da coluna lombar é necessário que ocorra rápida capacidade de produção de força para aumentar a rigidez do sistema formado pelos componentes osteomioarticulares desta região, bem como otimização da demanda energética e mobilidade (VAN DIEEN, CHOLEWICKI \& RADEBOLD, 2003; REEVES, NARENDRA \& CHOLEWICKI, 2007). Na maioria das atividades cotidianas, essas demandas de rigidez e mobilidade devem estar em equilíbrio (RADEBOLD et al., 2000). Nesse sentido, a coativação dos músculos agonistas e antagonistas do tronco é essencial para evitar falência do sistema (PANJABI, 1992).

Atualmente, destacam-se as alterações do recrutamento dos músculos do tronco - o que reduz a estabilidade da coluna vertebral - como o principal fator causal da dor lombar inespecífica (NEWCOMER et al., 2002; VAN DIEEN, CHOLEWICKI \& RADEBOLD, 2003). Desse modo, vários programas de intervenção utilizando exercícios de estabilização segmentar da coluna foram propostos com 0 intuito de aprimorar a estabilidade lombar (AROKOSKI et al., 2004; MCGILL \& KARPOWICZ, 2009). No entanto, esses programas apresentam um alto custo, pois, em geral, necessitam de acompanhamento profissional e grande demanda de tempo (ANDERS, WENZEL \& SCHOLLE, 2007).

Uma opção para auxiliar na automatização de padrões de ativação dos músculos do tronco são os exercícios realizados com hastes oscilatórias (ANDERS, WENZEL \& SCHOLLE, 2007). Esse tipo de exercício permite uma grande quantidade de variações, auxilia a co-ativação da musculatura do tronco e cria um equilíbrio entre mobilidade e estabilidade (ANDERS, WENZEL \& SCHOLLE, 2008). Moreside, Vera-Garcia e McGill (2007) analisaram o padrão de ativação dos músculos do tronco durante exercícios com haste oscilatória e identificaram que o modo como o exercício é executado pode interferir na estabilidade da coluna. Além disso, Anders, Wenzel e Scholle (2008) e Sánchez-Zuriága, Vera-Garcia e Moreside et al. (2009) identificaram que o plano de oscilação da haste e a postura na qual o exercício é executado podem modificar a ativação dos músculos do tronco. No entanto, nestes estudos, a comparação do efeito da execução de exercícios unilateralmente e bilateralmente, bem como as respostas de ativação muscular frente a esse tipo de exercícios em indivíduos do gênero feminino são questões inexploradas na literatura.
Dessa forma, o presente estudo teve por objetivo comparar a co-ativação dos músculos do tronco frente a diferentes exercícios realizados com uma haste oscilatória nas posturas em pé e sentada. Assim, hipotetiza-se que os exercícios realizados unilateralmente e na posição em pé causem maior ativação da musculatura estabilizadora do tronco.

\section{Método}

\section{Participantes}

Participaram do estudo doze mulheres com idade de 20,4 $\pm 1,9$ anos, destras, saudáveis e sem histórico de lesão ou dor músculoesquelética nos membros superiores e coluna lombar durante os seis meses pregressos ao estudo. As participantes assinaram o termo de consentimento livre e esclarecido e o estudo foi aprovado pelo comitê de ética local (no. 3156).

\section{Protocolo}

O protocolo de coleta de dados foi realizado em dois dias com intervalo mínimo de 24 e máximo de 72 horas entre cada dia. No primeiro dia, as participantes foram familiarizadas com o uso da haste oscilatória em todos os exercícios realizados durante a coleta de dados. No segundo dia, as participantes realizaram os exercícios com uma haste oscilatória (Flexibar $\left.{ }^{\circledR}\right)$ que possuía massa de $800 \mathrm{~g}$ e comprimento $150 \mathrm{~cm}$.

Três exercícios com a haste oscilatória comumente usados no treinamento e reabilitação muscular - foram selecionados para o estudo. $O$ exercício I foi executado bimanualmente com os ombros em aproximadamente $90^{\circ}$ de flexão e oscilação da haste no plano transversal, paralela ao solo. O exercício II também foi executado bimanualmente com os ombros em aproximadamente $180^{\circ}$ de flexão e oscilação da haste no plano frontal, paralela ao solo. $O$ exercício III foi executado com o membro superior dominante, ombros em aproximadamente $90^{\circ}$ de flexão e oscilação da haste no plano sagital, perpendicular ao solo (Figura 1).

Todos os exercícios foram realizados por 15 segundos, com um período de 60 segundos após cada exercício. O ritmo de movimentação da haste e do membro superior foi controlado por um metrônomo ajustado em $5 \mathrm{~Hz}$ (300 bpm). Esta frequência de movimentação da haste foi selecionada em estudo piloto por gerar menos movimentos acessórios e melhor coordenação do movimento. Sendo assim, foi considerada a 
frequência de movimentação mais próxima da frequência natural da haste (MORESIDE, VERAGARCIA \& MCGILL, 2008). A movimentação da haste foi, primordialmente, mantida pela flexão e extensão do cotovelo e as voluntárias foram orientadas a manter a movimentação em apenas um plano.
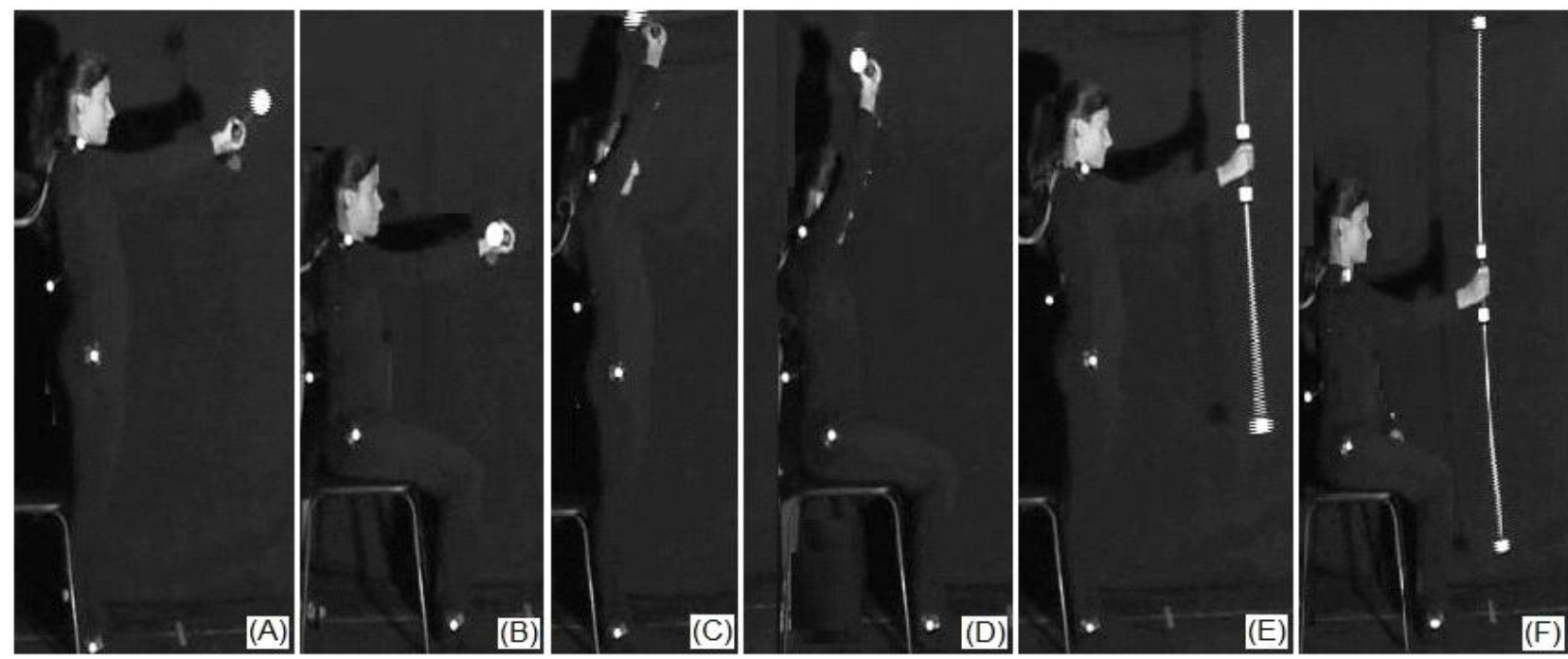

Figura 1. Posicionamento durante os exercícios: (A) exercício I, posição em pé; (B) exercício I, posição sentada; (C) exercício II, posição em pé; (D) exercício II, posição sentada; (E) exercício III, posição em pé; (F) exercício III, posição sentada.

Cada exercício foi realizado nas posições em pé e sentada na postura neutra, que foi caracterizada pelo ângulo de $180 \pm 15^{\circ}$ entre os pontos anatômicos do acrômio, trocanter maior do fêmur e maléolo lateral, na posição em pé, e ângulo de $135 \pm 15^{\circ}$ entre acrômio, trocanter maior do fêmur e processo espinhoso de $L 1$, na posição sentada (O'SULLIVAN et al., 2006). Para os exercícios na posição sentada, uma cadeira sem encosto e com altura ajustável foi utilizada. A postura neutra na posição em pé e sentada foi adotada no início dos exercícios, e as participantes foram instruídas a manter o correto alinhamento dos marcadores localizados no acrômio, quadril e tornozelo, na posição em pé e acrômio, quadril e coluna, na posição sentada (O'SULLIVAN et al., 2006). O feedback visual do posicionamento das marcas no plano sagital foi fornecido para as voluntárias por meio de um monitor posicionado à frente destas.

\section{Cinemetria}

Para coleta dos dados de cinemetria foram utilizados marcadores fotoreflexivos posicionados sobre os pontos anatômicos do acrômio, trocanter maior do fêmur, processo espinhoso de L1 e maléolo lateral, bem como, uma câmera (Panasonic $^{\circledR}$ ) com frequência de captura de imagens de trinta quadros por segundo, que foi posicionada no plano sagital em uma distância de dois metros do voluntário e em uma altura referente a $50 \%$ da estatura do participante. Além disso, foram posicionados na haste oscilatória marcadores fotoreflexivos sobre as extremidades da haste. Os dados de cinemetria foram coletados pelo software MRXP 1.07 (Noraxon $^{\circledR}$ ) em sincronia com os dados de EMG. Além disso, para a determinação do início e final de cada exercício foi utilizado um sinal eletro-luminoso coletado também pelo software MRXP 1.07 $\left(\right.$ Noraxon $\left.^{\circledR}\right)$.

Para a análise dos dados de cinemetria foi utilizado o software Vicon Peak 9.0 (Peak Motus ${ }^{\circledR}$ ) no qual foi calculada a frequência de oscilação da haste e angulação da postura durante os exercícios.

\section{Eletromiografia}

A coleta do sinal de EMG foi realizada com a utilização de eletrodos de superfície de $\mathrm{Ag} / \mathrm{AgCl}$ $\left(\right.$ Meditrace $^{\circledR}$ ) com área de captação ativa de $1 \mathrm{~cm}^{2}$ e distância intereletrodos de $2 \mathrm{~cm}$ em configuração bipolar. Os eletrodos bipolares foram posicionados no lado direito sobre os músculos: oblíquo interno $(\mathrm{Ol}$; em $2 \mathrm{~cm}$ medialmente e inferiormente da espinha ilíaca ântero-posterior), oblíquo externo (OE; em 50\% da distância entre a região inferior da caixa torácica e espinha ilíaca ântero-superior), reto abdominal (RA; em $1 \mathrm{~cm}$ abaixo da cicatriz umbilical e $2 \mathrm{~cm}$ da linha média), iliocostal lombar (IL; em $6 \mathrm{~cm}$ lateralmente ao espaço entre o 
processo espinhoso de L2-L3; e multifido (MU; em $2 \mathrm{~cm}$ lateralmente ao espaço entre o processo espinhoso de L4-L5) (MARCUCCI et al., 2003; MARSHALL \& MURPHY, 2003; O'SULLIVAN et al., 2006). O eletrodo de referência foi posicionado sobre o acrômio direito. Antes da colocação dos eletrodos foi realizada a tricotomia, abrasão com lixa fina e limpeza com álcool (GONCCALVES \& BARBOSA, 2005).

Um módulo de aquisição de sinais biológicos por telemetria de 16 canais (Noraxon®) e 0 software de aquisição de dados deste equipamento MRXP 1.07 software (Noraxon $\AA$ ) com frequência de amostragem de 1000 amostras por segundo com ganho total de 2000 vezes $(20$ no pré-amplificador e 100 no amplificador) foi utilizado para a coleta do sinal de EMG.

\section{Análise dos dados}

A análise do sinal EMG foi realizada utilizando rotinas desenvolvidas em ambiente Matlab. Para o processamento do sinal foram utilizados: filtro passa alta de $20 \mathrm{~Hz}$ e passa baixa de $500 \mathrm{~Hz}$. $\mathrm{O}$ sinal de EMG foi analisado no domínio do tempo para o cálculo dos valores de Root Mean Square (RMS) em janelas móveis de 250 ms com overlap de $125 \mathrm{~ms}$ para análise da atividade muscular

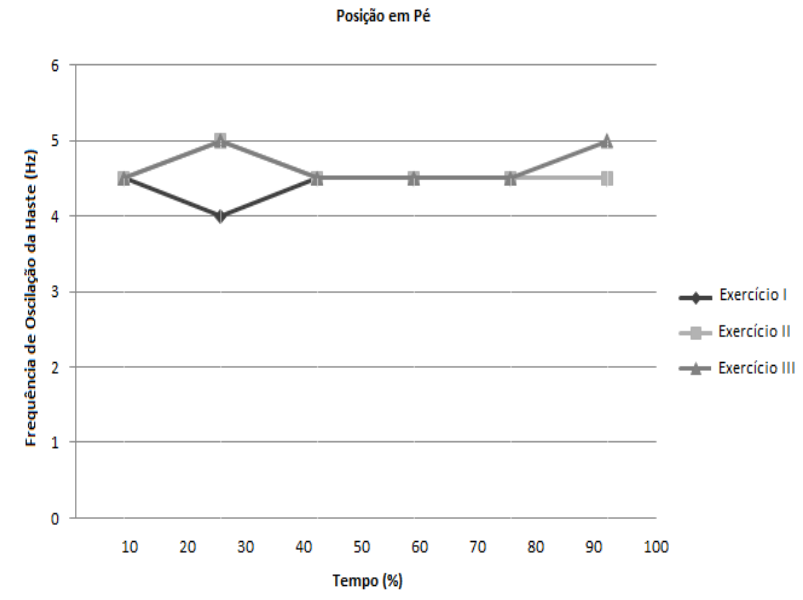

durante todo o período de exercícios (15 segundos). $O$ padrão de co-ativação dos músculos do tronco foram analisados utilizando as seguintes razões com os valores da amplitude do sinal: ântero-posterior $(\mathrm{A} / \mathrm{P}=\mathrm{RA}+\mathrm{EO}+\mathrm{IO} / \mathrm{IL}+\mathrm{MU}), \mathrm{MU} / \mathrm{LL}$ e OI/OE .

\section{Análise estatística dos dados}

Para a análise estatística foi utilizado um único valor médio de RMS de cada voluntária em cada condição testada. Assim, a análise estatística foi realizada por meio do software PASW Statistic $18.0\left(\right.$ SPSS $\left.^{\circledR}\right)$ utilizando para as comparações os testes ANOVA para medidas repetidas e post-hoc de Bonferroni, sendo adotado o nível de significância quando $p<0,05$.

\section{Resultados}

Frequência de oscilação da haste oscilatória e posicionamento angular da postura das voluntárias durante os exercícios

A frequência de oscilação da haste foi mantida em aproximadamente $4 \pm 0,1 \mathrm{~Hz}$ (Figura 2).

A postura das voluntárias durante os exercícios realizados em pé foi de $172 \pm 6,3^{\circ}$ e para os exercícios realizados em postura sentada foi de $134 \pm 10,5^{\circ}$ (Tabela 1).

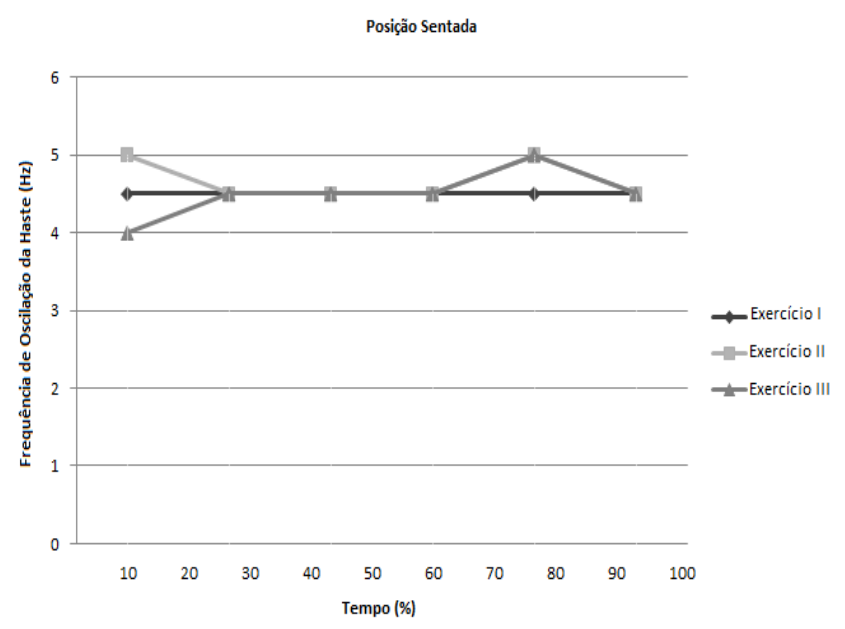

Figura 2. Frequência da oscilação da haste durante os exercícios I, II e III.

Tabela 1. Posicionamento angular da postura das participantes durante os exercícios I, II e III nas posições em pé e sentada.

\begin{tabular}{ccc}
\hline & \multicolumn{2}{c}{ Postura () } \\
Exercício I & Posição em Pé & Posição Sentada \\
\cline { 2 - 3 } Exercício II & $176,12 \pm 3,1$ & $134,5 \pm 6,1$ \\
Exercício III & $165,65 \pm 1,7$ & $124 \pm 9,8$ \\
Total & $177,10 \pm 0,3$ & $145,1 \pm 9,6$ \\
\hline
\end{tabular}

Comparação da ativação muscular entre os tipos de exercício e postura

Para a razão A/P os exercícios I e \|| apresentaram maior valor em relação ao exercício III ( $p=0,00$ e $p=0,00)$, bem como, a razão $A / P$ foi maior na postura sentada em relação à postura em pé $(p=0,00)$ (Figura 3 e Figura 4).

Para a razão MU/IL, o valor desta foi maior no exercício II em relação ao exercício III $(p=0,00)$ e 
maior na posição sentada em relação à posição em pé $(p=0,03)$ (Figura 3 e Figura 4$)$.

Os exercícios II e III apresentaram maiores valores de razão OI/OE em relação ao exercício I $(\mathrm{p}=0,00$ e $\mathrm{p}=0,00)$, assim como, os exercícios realizados na postura sentada tiveram valores maiores desta razão do que quando foram realizados na postura em pé $(p=0,00)$ (Figura 3 e Figura 4).

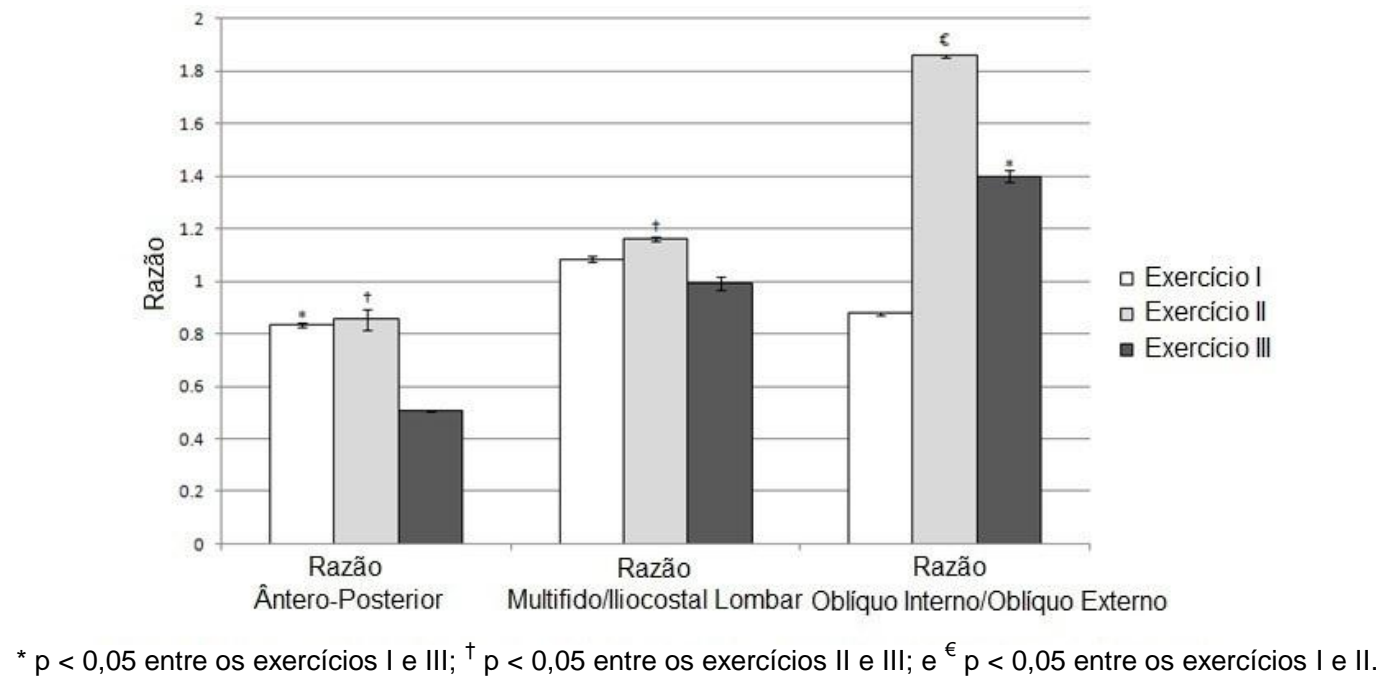

Figura 3. Comparação entre os valores das razões ântero/posterior, multifido/iliocostal lombar e oblíquo externo nos exercícios realizados com a haste oscilatória.

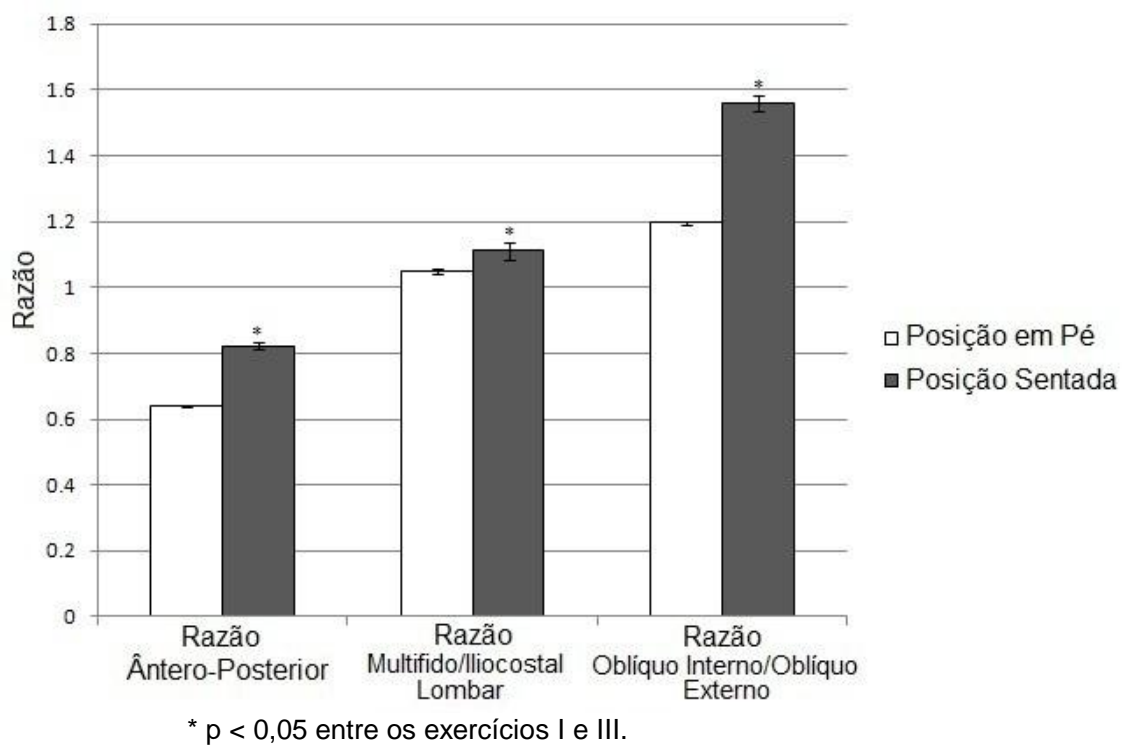

Figura 4. Comparação entre os valores das razões ântero/posterior, multifidus/iliocostal lombar e oblíquo interno/oblíquo externo nos exercícios realizados com a haste oscilatória.

\section{Discussão}

A literatura científica aponta duas diferentes vertentes para o entendimento da estabilidade da coluna lombar. Uma dessas teorias afirma que um único grupo de músculos (locais) possui exclusiva função de prover a estabilidade, enquanto o outro grupo de músculos (globais) é exclusivamente responsável por gerar torque e movimento do tronco. Conforme esta classificação, os músculos locais, representados pelo $\mathrm{MU}$ e OI, deveriam apresentar atividade eletromiográfica referente a uma contração isométrica frente à ocorrência de perturbações cinemáticas externas. Enquanto os músculos globais, representados pelos músculos IL, RA e $\mathrm{OE}$, deveriam apresentar um comportamento do sinal eletromiográfico isotônico (ANDERS, WENZEL \& SCHOLLE, 2008). Contudo, O comportamento do sinal de EMG dos músculos do tronco durante exercícios com haste oscilatória não apresentou este padrão esperado. 
Neste sentido, os achados do presente estudo estão em acordo com a outra teoria, que preconiza que a direção das forças externas desestabilizadoras são os fatores que irão definir qual o grupo muscular que será recrutado (CHOLEWICKI \& MCGILL, 1996; CHOLEWICKI \& VANVLIET, 2002; PAÑEGO et al., 2009). Nos achados do presente estudo foi possível identificar que o tipo de exercício, caracterizado pelo plano de oscilação da haste, e a postura foram determinantes para a definição do padrão de co-ativação muscular.

Os resultados demonstraram que houve um predomínio na atividade eletromiográfica dos músculos da região posterior em relação aos músculos da região anterior do tronco. Essa maior ativação dos músculos da região dorsal pode estar relacionada ao fato de, para o exercício I, estes músculos possuírem maior ativação com o intuito de reduzir o deslocamento anterior do centro de gravidade provocado pela oscilação da haste à frente do tronco. No exercício II, devido ao posicionamento dos membros superiores, há uma elevação da caixa torácica durante a movimentação da haste, o que também pode ter corroborado para 0 maior valor da razão $A / P$ (PAÑEGO et al., 2009).

Notoriamente, a co-ativação dos músculos MU e Ol é fundamental para aumentar a estabilidade da coluna lombar. O MU é o único músculo capaz de aumentar a estabilidade dos segmentos vertebrais lombares enquanto estabiliza dinamicamente a articulação sacro-ilíaca (SÁNCHEZ-ZURIÁGA et al., 2009). A região ântero-inferior do abdôme, formada pelo transverso abdominal e o Ol, estabiliza a coluna por meio da fáscia tóraco-lombar, bem como aumenta a pressão intra-abdominal, o que eleva a estabilidade da articulação sacro-ilíaca (O'SULLIVAN et al., 2002; O'SULLIVAN et al., 2006).

Para a razão MU/IL, o exercício II apresentou maior valor em relação aos exercícios I e III. Tal fato pode estar relacionado com a mudança do plano de oscilação da haste que, neste caso, resulta em ação fásica do MU (ANDERS, WENZEL \& SCHOLLE, 2008). Assim, em acordo com os achados de Andres et al. (2008), as mudanças no plano de ativação observadas no nosso estudo geram mudanças nas características do padrão de ativação dos músculos posteriores (ANDERS, WENZEL \& SCHOLLE, 2007).
Para a razão OI/OE, nos exercícios 2 e 3, foi observada maior ativação do músculo OI. Segundo Sánchez-Zuriága, Vera-Garcia e Moreside et al. (2009), que compararam os efeitos de diferentes planos de oscilação em exercícios com haste oscilatória, os músculos OI e OE aparentemente respondem com uma maior ativação às perturbações verticais, típicas no exercício II. Nesse tipo de exercício, esta maior co-ativação dos músculos oblíquos ocorre na tentativa de estabilizar a coluna e reduzir a rotação axial do tronco (BOGDUK, 1997). Esse mesmo padrão de co-ativação do OI e OE pode ocorrer quando se realiza 0 exercício unilateralmente, como no caso do exercício III, pois, além de evitar o deslocamento anterior do tronco, estes músculos estabilizam o segmento evitando movimentos acessórios de rotação (KAPANDJI, 1995; KENDALL, KENDALL \& PROVANCE, 1995; SNIJDERS et al.; 1995).

A postura em que o exercício é realizado modifica a co-ativação muscular, sendo que na postura em pé ocorre maior demanda de atividade dos músculos IL e OE. Isto ocorre porque na condição sentada, o tronco tende a sofrer reduzida perturbação oscilatória, comparado a postura em pé, uma vez que a pelve encontra-se mais estável devido à redução da ação dos músculos flexores do quadril. Contudo, no presente estudo não foi realizada a coleta do sinal eletromiográfico de músculos flexores do quadril, tal como o reto femoral, o que poderia proporcionar informações mais precisas acerca da atuação dos músculos desta região durante exercícios realizados com haste oscilatória nas posições em pé e sentada.

\section{Conclusão}

O padrão de recrutamento muscular durante exercícios com haste oscilatória varia de acordo com o plano de oscilação da haste. Sendo que foi possível verificar que nos exercícios realizados com a haste oscilando no plano sagital e frontal ocorre uma maior co-ativação dos músculos oblíquos. Além disso, o fato do exercício III ter sido executado, unilateralmente, pode ter corroborado para a maior razão Ol/OE. Portanto, a estabilidade da coluna não é essencialmente realizada por um único grupo de músculos do tronco, mas depende da ação conjunta de todos os músculos dessa região, sendo que, as direções das forças desestabilizadoras determinarão qual músculo será mais ativo. 


\section{Referências}

ANDERS, C.; WENZEL, B.; SCHOLLE, H. C. Cyclic upper body perturbations caused by a flexible pole: influence of oscillation frequency and direction on trunk muscle co-ordination. Journal of Back and Musculoskeletal Rehabilitation, Amsterdam, v. 20, p. 167-175, 2007.

ANDERS, C.; WENZEL, B.; SCHOLLE, H. C. Activation characteristics of trunk muscles during cyclic upper-body perturbations caused by an oscillating pole. Archives of Physical Medicine and Rehabilitation, New York, v. 89, p. 13141322, 2008.

AROKOSKI, J. P.; VALTA, T.; KANKAANPÄÄ, M.; AIRAKSINEN, O. Activation of lumbar paraspinal and abdominal muscles during therapeutic exercises in chronic low back pain. Archives of Phyisical Medicine and Rehabilitation, New York, v. 85, p. 823-832, 2004.

BOGDUK, N. The lumbar muscles and their fascia. In: BOGDUK N. Clinical anatomy of the lumbar spine and sacrum. 3. ed. London: Churchill Livingstone, 1997. P. 101-125.

CHOLEWICKI, J.; MCGILL, S. Mechanical stability of the in vivo lumbar spine: implications for injury and chronic low back pain. Clinical Biomechics, Bristol, v. 11, p. 1-15, 1996.

CHOLEWICKI, J.; VANVLIET, J. I. V. Relative contribution of trunk muscles to the stability of the lumbar spine during isometric exertions. Clinical Biomechanics, Bristol, v. 17, p. 99-105, 2002.

GRANATA, K. P.; MARRAS, W. S. Cost-benefit of muscle cocontraction in protecting against spinal instability. Spine, Lebanom, v. 25, p. 1398-1404, 2000.

GONÇALVES, M.; BARBOSA, F. Análise de parâmetros de força e resistência dos músculos eretores da espinha lombar durante a realização de exercício isométrico em diferentes níveis de esforço. Revista Brasileira de Medicina do Esporte, São Paulo, v. 11, p. 102-114, 2005.

KAPANDJI, A. I. Fisiologia articular: tronco e coluna vertebral. 5. ed. Rio de Janeiro:

Guanabara Koogan, 2000.

KENDALL, F. P.; KENDALL, E.; PROVANCE, P. G. Músculos provas e funções. 4. ed. São Paulo: Manole, 1995.

MACDONALD, D.; MOSELEY, G. L.; HODGES, P. W. Why do some patients keep hurting their back? evidence ongoing back muscle dysfunction during remission from recurrence back pain. Pain, Seattle, v. 142, p. 183-188, 2009.
MARCUCCI, F. C. I.; CARDOSO, N. S.; BERTELLI, K. S.; GARANHI, M. R.; CARDOSO, J. R. Alterações eletromiográficas dos músculos do tronco de pacientes com hemiparesia após acidente vascular encefálico. Arquivos de Neuropsiquiatria. São Paulo, v. 65, p. 900-905, 2007.

MARSHALL, P.; MURPHY, B. The validity and reliability of surface EMG to assess the neuromuscular response of the abdominal muscles to rapid limb movement. Journal of Electromyography and Kinesiology. New York, v. 13, p. 477-489, 2003.

MCGILL, S.; KARPOWICZ, A. Exercise for spine stabilization: motion /motor patterns, stability progressions, and clinical technique. Archives of Phyisical Medicine and Rehabilitation. New York, v. 90, p. 118-126, 2009.

MORESIDE, J. M.; VERA-GARCIA, F. J.; MCGILL, S. M. Trunk muscle activation patterns, lumbar compressive forces, an spine stability when using the Bodyblade. Physical Therapy Journal. Jersey City, v. 87, p. 153-164, 2007.

NEWCOMER, K. L; JACOBSON, T. D.; GABRIEL, D. A.; LARSON, D. R.; BREY, R. H.; AN, K. N. Muscle activation patterns in subjects with and without low back pain. Archives of Physical Medicine and Rehabilitation. New York, v. 83, p. 816-821, 2002.

OLIVEIRA, A. S. C.; GONÇALVES, M. Lumbar muscle recruitment during resistance exercise for upper limbs. Journal of Electromyography and Kinesiology. New York, v. 19, p. 737-745, 2009.

O'SULLIVAN, P.; DANKAERTS, W.; BURNETT, A. F.; FARREL, G. T.; JEFFORD, E.; NAYLOR, C. $S$. Effect of different upright postures on spinalpelvic curvature and trunk muscle activation in a pain free population. Spine, Lebanom, v. 31, p. E707-712, 2006.

O'SULLIVAN, P. B.; GRAHAMSLAW, K. M.; KENDELL, M.; LAPENSKIE, S. C.; MÖLER, N. E.; RICHARDS, K. V. The effects of different standing and sitting postures on trunk muscle activity in a pain free population. Spine, Lebanom, v. 27, p. 1238-1244, 2002.

O'SULLIVAN, P.; TWOMEY, L.; ALLISON, B.; SINCLAIR, J.; MILLER, K.; KNOX, J. Altered patterns of abdominal muscle activation in patients with chronic low back pain. Australian Journal of Physiotherapy, Sidney, v. 43, p. 91 98, 1997.

PAÑEGO, M. M.; VERA-GARCÍA, F. J.; SÁNCHEZ-ZURIAGA, D.; SARTI-MARTÍNEZ, M. A. Electromyographic studies in abdominal 
exercises: a literature synthesis. Journal of Manipulative and Physiological Therapeutics, Lombardi, v. 32, p. 232-242, 2009.

PANJABI, M. M. The stabilizing system of the spine: part I: function, dysfunction, adaptation, and enhancement. Journal of Spinal Disorder, Kansas City, v. 5, p. 383-389, 1992.

RADEBOLD, A.; CHOLEWICKI, J.; PANJABI, M. M.; PATEL, T. C. Muscle response pattern to sudden trunk loading in healthy individuals and in patients with chronic low back pain. Spine, Lebanom, v. 25, p. 947-954, 2000.

REEVES, N. P.; NARENDRA, K. S.; CHOLEWICKI, J. Spine stability: six blind men and the elephant. Clinical Biomechanics, Bristol, v. 22, p. 266-274, 2007.

SÁNCHEZ-ZURIÁGA, D.; VERA-GARCIA, F. J.; MORESIDE, J. M. Trunk muscle activatin patterns and spine kinematics when using an oscillating blade: influence of different postures and blade orientation. Archives of Physical Medicine and Rehabilitation, New York, v. 90, p. 1055-1060, 2009.

SNIJDERS, C. J.; BAKKER, M. P.; VLEEMING, A.; STOECKART, R.; STAM, H. J. Oblique abdominal muscle activity in standing and sitting on hard and soft seats. Clinical Biomechanics, Bristol, v. 10, p. 73-78, 1995.

VAN DIEEN, J. H.; CHOLEWICKI, J.;

RADEBOLD, A. Trunk muscle recruitment patterns in patients with low back enhance the stability of lumbar spine. Spine, Lebanom, v. 28, p. 834-841, 2003.
Endereço:

Mauro Gonçalves

Unesp - Laboratório de Biomecânica

Avenida 24-A, 1515 Bela Vista

Rio Claro SP Brasil

13506-900

Telefone: (19) 3526-4345

e-mail: labiomec@rc.unesp.br 